\title{
Patterns of Asian and non-Asian morbidity in hospitals
}

\author{
L J DONALDSON, J B TAYLOR
}

\begin{abstract}
On the basis of surname, 6418 Asians were identified out of a total of 109187 deaths and discharges of Leicestershire residents who had been treated in hospitals in the Trent Regional Health Authority over two years. After linkage to Hospital Activity Analysis computerised records, hospital morbidity in Asians and non-Asians was compared. Asian patients in certain age groups were more likely than non-Asian patients to be diagnosed as having asthma; leukaemia; diabetes mellitus; blood, thyroid, and eye disorders; certain forms of heart disease; and spontaneous and other types of abortion (excluding therapeutic abortion). The well recognised excess of cases of tuberculosis among Asians was also confirmed. Conversely, for some age groups, Asians were less likely than non-Asians to fall into particular diagnostic categories. Exploring such apparent differences can serve as the basis for aetiological inquiry and service planning. The patient's name is now probably the most reliable indicator of Asian ethnic origin in health records.
\end{abstract}

\section{Introduction}

Until recently research workers investigating the health of immigrant populations in Britain have focused on traditional, known associations between ethnic origin and disease, particu-

\footnotetext{
Department of Community Health, Leicester Royal Infirmary, Leicester LE2 7LX

L J DONALDSON, MD, FRCSED, senior lecturer in epidemiology, Leicester University

J B TAYLOR, BSC, MD, statistician, Leicestershire Health Authority

Correspondence and requests for reprints to: Dr L J Donaldson.
}

larly infectious ${ }^{12}$ and nutritional ${ }^{3}$ diseases. A recent symposium in Birmingham, ${ }^{4}$ however, has shown that a broader range of conditions, including chronic diseases, are being studied. In other countries where large immigrant communities exist epidemiological studies have compared patterns of mortality and morbidity in these populations with the indigenous population and in some cases with those in the migrants' country of origin. An early example was the study of mortality from cardiovascular diseases among the Japanese in the United States, Hawaii, and Japan. ${ }^{5}$ If such studies can show that differences in the frequency of disease are real and not artefactual firstly they may give clues for aetiological inquiry and, secondly, they may suggest scope for initiatives in the Health Service.

Studies using data that are routinely available, such as Hospital Activity Analysis (HAA), are hampered because valid recording of ethnic group is not done on health records. Reliance on information collected about place of birth is hazardous because such data may be incomplete and do not in any case apply to immigrants born in Britain. ${ }^{6}$ ?

Our study, which compares the pattern of hospital morbidity in all Asian and non-Asian patients in a defined geographical area over two years, has included all age groups and attempted to surmount the problem of missing data by classifying patients of Asian ethnic origin by their names.

\section{Methods}

The starting point for the investigation was a list of the surnames of all 109187 Leicestershire residents who died in, or were discharged from, any National Health Service hospital (other than psychiatric or maternity) in the Trent Regional Health Authority during 1977 and 1978.

Asian survey workers picked out 6418 names of Asian appearance from this list. This is considered to be a more reliable method of identifying a person of Asian origin than using country of birth. ${ }^{7}$ The meaning of "Asian origin" in this study is a person not of United Kingdom descent who originates from India, Pakistan, or Bangladesh or a person of Indian and Pakistani descent who originates from east 


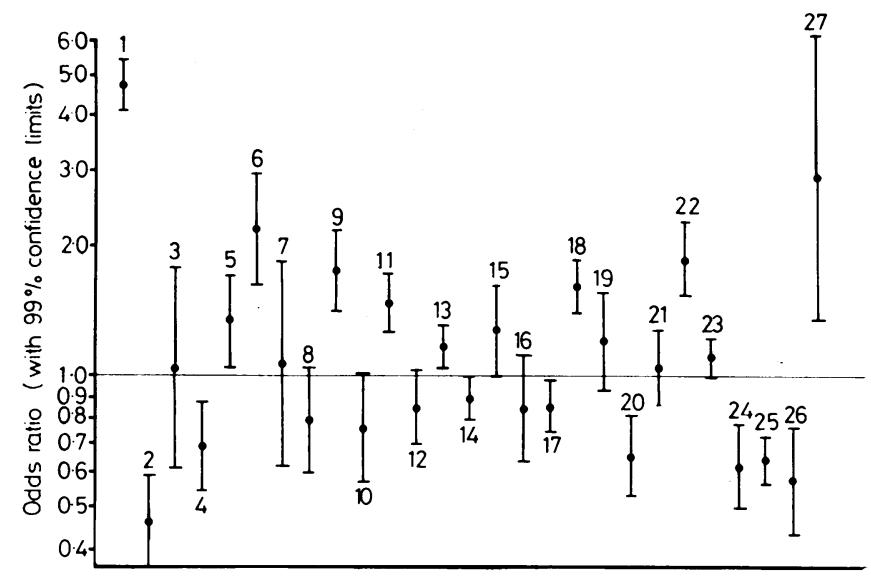

Diagnostic category

Ratio of odds (with $99 \%$ confidence limits) of Asians being discharged from hospital in a particular diagnostic category compared with non-Asians. 1 Infective and parasitic diseases (ICD No 000-136), 2 all malignant neoplasms (140-199), 3 neoplasms of lymphatic and haematopoietic tissues (200-209), 4 benign neoplasms and neoplasms of unspecified nature (210-239), 5 endocrine, nutritional, and metabolic diseases (240-279), 6 diseases of blood and blood forming organs (280-289), 7 mental disorders (290-315), 8 diseases of nervous system (320-358), 9 diseases of the eye (360-379), 10 diseases of the ear and mastoid process (380-389), 11 rheumatic fever, hypertensive disease, and heart disease (390-429), 12 diseases of peripheral circulatory system (430-458), 13 diseases of respiratory system $(460-519), 14$ diseases of digestive system (520-577), 15 diseases of urinary system (580-599), 16 male genital disorders (600-607), 17 diseases of breast and female genital system (610-629), 18 conditions of pregnancy, childbirth, and puerperium (630-678, Y60-Y61), 19 diseases of skin and subcutaneous tissue (680-709), 20 diseases of musculoskeletal system and connective tissue (710-738), 21 congenital anomalies (740-759), 22 certain causes of perinatal morbidity (760-779), 23 symptoms and ill-defined conditions (780-796), 24 fractures, dislocations, and sprains (800-848), 25 other injuries and reactions (850-999), 26 people undergoing preventive procedures (Y40-43, Y45, Y $50, \mathrm{Y} 52$, Y62-79), and 27 unclassified.

Africa. Place of birth is one of the items recorded on the Hospital Activity Analysis source document in the Trent region. An analysis of country of birth of the Asians identified in this study confirms the document's unreliability as a potential indicator of ethnic origin. It showed proportions as follows: $45 \%$ United Kingdom; $31 \%$ India, Pakistan, or Bangladesh; $19 \%$ east Africa; $5 \%$ other countries. The possibility, through the use of names, of serious under enumeration of Asian women by this method due to cross cultural marriage is unlikely because this is estimated to be low in Britain now. ${ }^{8}$ Once identified, the variable of Asian ethnic origin was added to the computer records of all relevant deaths and discharges held on the magnetic tapes of HAA data for all Leicestershire residents over the same two years. To preserve confidentiality names were removed from the HAA computer tape.

Questions on parents' country of birth, included in the 1971 census (which provided a crude estimate of the population of New Commonwealth origin) were not included in the 1981 census. $^{9}$ Valid estimates of the population of Asian ethnic origin at risk were therefore not available and thus it was not possible to compare discharge rates for non-Asian and Asian populations.

A comparative measure was chosen to indicate hospital morbidity in the two groups. The procedure essentially entailed the calculation of a series of odds ratios for each diagnostic group (X) and each age-sex group. The analysis took account of the number of patients falling into each of four possible categories: (a) Asians with diagnosis X; (b) Asians with diagnoses other than $\mathrm{X}$; (c) non-Asians with diagnosis $\mathrm{X}$; and $(d)$ non-Asians with diagnoses other than $\mathrm{X}$.

For the Asians, the odds in favour of being discharged with the particular diagnosis $X$ were therefore $a / b$; for the non-Asians the odds were $c / d$. The odds ratio was then: $a / b \div c / d=(a d / b c)$.

The $99 \%$ confidence limits for the odds ratios were calculated using the method originally described by Woolf ${ }^{10}$ (readers are referred to this paper for details and worked examples).

A value of unity for this odds ratio indicated a balance between Asian and non-Asian discharge ratios. A value greater than unity suggested that Asian patients may be more likely to be discharged with condition $\mathrm{X}$ than non-Asian patients, though due consideration must be given to the confidence interval. A value less than unity for the odds ratio $D$ suggests that Asian patients may be less likely to be discharged with condition $\mathrm{X}$ than non-Asian patients.

\section{Results}

The figure shows odds ratios for the major disease groupings used $\mathbb{D}_{\mathbb{D}}$ in the " $A$ " list categorisation of the International Classification of Diseases, 8th revision. ${ }^{11}$ The ratios have been weighted, using Woolf's method, to take account of the fact that individual age groups contribute to differing extents.

The highest ratio was shown for infectious and parasitic diseases, $\stackrel{\mathcal{O}}{\mathscr{T}}$ when the odds of being discharged with this diagnosis were five times greater in Asians than in non-Asians. Taking account of both the estimate of the odds ratio and its $99 \%$ confidence limits, the figure $\frac{\omega}{\vec{D}}$ shows that unequivocally greater Asian morbidity was also observed $\stackrel{\odot}{\triangle}$ for endocrine, nutritional, and metabolic diseases; disorders of the blood; diseases of the eye; heart disease; respiratory disease; condi- $\infty$ tions of pregnancy, childbirth, and the puerperium; perinatal $\vec{O}$ morbidity; and the unclassifiable disease category, although here (as $\overrightarrow{-}$ indicated by the width of the confidence limit) the numbers were very $\omega$ small. Conversely, unequivocally lower Asian morbidity was observed $\frac{\partial}{\partial}$ for benign and malignant neoplasms; diseases of the musculoskeletal system and connective tissue; fractures and other injuries; and conditions where preventive procedures were undertaken. A more detailed examination of diagnostic groups within individual age os groups showed more fully the differences between the two ethnic $\mathscr{G}^{\circ}$ groups.

The high odds ratio for infectious and parasitic diseases was accounted for by differences for tuberculosis, where ratios were high 6 for all age groups above the age of 5 years for both the pulmonary and the non-pulmonary forms. Ratios for non-pulmonary tuberculosis $\overrightarrow{\vec{\varphi}}$ were slightly higher than those for pulmonary tuberculosis in all age $\varphi$ groups except 5-14 year olds. The table shows the odds ratios and $99 \%$ confidence limits for conditions other than infectious and para- $\overline{3}$ sitic diseases in which the ratios were either unequivocally high or unequivocally low.

Ratio of odds of Asian patients being discharged from hospital with a particular diagnosis* compared with non-Asians to show diagnostic categories and age groups where values were unequivocally high or low

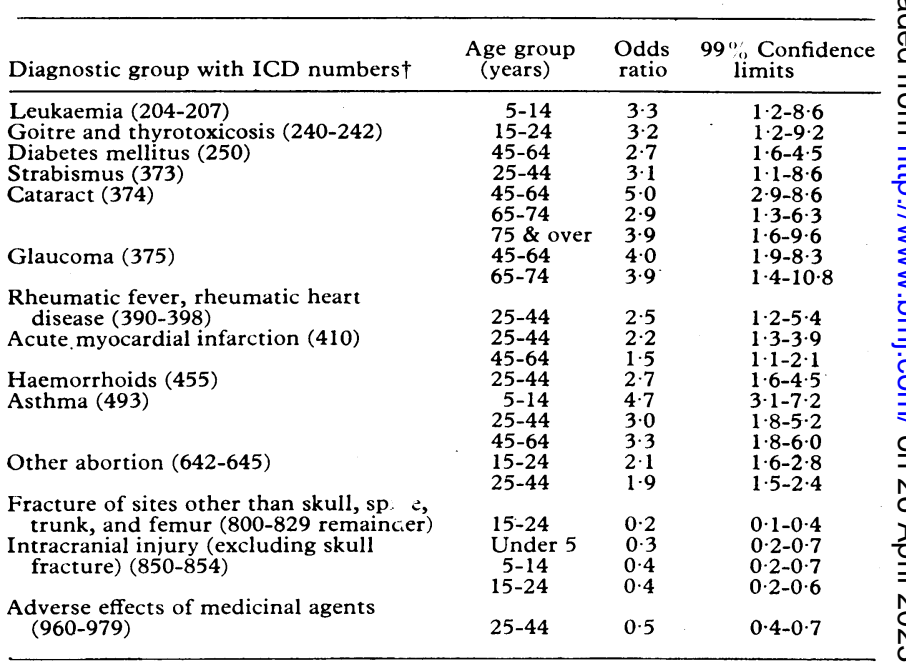

* Infectious and parasitic diseases have been excluded.

+8 th revision of the International Classification of Diseases."

\section{Discussion}

Methodological pitfalls are common to all studies of hospital morbidity. The actual proportion of sufferers of a particular disease treated as inpatients will be influenced by a complex $\stackrel{\Phi}{\varrho}$ interaction of factors, including patients' perceptions of ill $\bar{q}$ health, referral patterns, availability of resources, and styles of $\delta$ hospital medical practice. Furthermore, HAA data are based on events (deaths and discharges) not patients so that, for example, a patient readmitted or transferred during the study period would 
appear as two apparently separate records. Here, these limitations would be important only as explanations of apparent differences in morbidity between Asian and non-Asians if they affected the two groups to different extents.

Aside from the potentially spurious explanations of disease associations in which there is an apparent excess for the Asian patients, two interpretations are possible. Firstly, a residue of certain diseases in the Asian population becomes apparent after immigration because of availability and access to services that may not be easily obtainable in the country of origin. Of the diseases shown in the table, eye disorders (strabismus, cataract, and glaucoma) and diabetes mellitus might fall into this category. Secondly, the Asian population is at greater risk of contracting another group of conditions because of host or environmental factors including life style. In the former group the implications are mainly for the provision of services while in the latter there may also be scope for aetiological inquiry.

The observation of a raised odds ratio for acute myocardial infarction is in line with other studies that have shown higher mortality and higher rates of hospital admission, ${ }^{12}{ }^{13}$ although no hypothesis has been put forward to explain these differences. The apparent excess of asthma in the younger Asian age groups could reflect greater host susceptibility to environmental factors, although others reporting on ethnic differences in childhood asthma suggested that admission rates may be higher for immigrant groups. ${ }^{14}$ The number of deaths from asthma in hospital was very low overall but there appeared to be no difference between the two ethnic groups.

The presence of only small numbers of cases precluded finer diagnostic breakdown of blood disorders, thyroid disease, and the distinction between rheumatic fever and rheumatic heart disease.

The excess in the "other abortion" category that includes spontaneous abortion agrees with reports of excess perinatal mortality in Asian mothers ${ }^{15}$ and may represent part of a range of increased fetal loss in this ethnic group.

The observation of an apparently higher frequency of leukaemia for Asians compared with non-Asians in the 5-14 year old age group was based on relatively small numbers of discharges (nine Asians, 36 non-Asians). The possibility that the difference was explained by a differential readmission rate between the two ethnic groups was tested for this small body of data by examining the number of discharges per person, and there was no apparent discrepancy between Asians and nonAsians. Therefore, if this finding is not another artefact arising from the data or due to selective immigration it suggests an area of further investigation.

Indeed, the purpose of a descriptive epidemiological study, whether based on mortality data, hospital inpatient data, or other sources of morbidity data, is to generate hypotheses that are then tested by studies designed to include the collection of data from individuals. In investigating risk factors and disease in Asians living in Britain even descriptive studies are made difficult because valid recording of ethnic group is not done on many health records. Nor is the ethnic group included in population census data to allow the construction of reliable denominators. Furthermore, when moving to formal study design, obtaining and gaining access to valid sampling frames for the Asian population is likely to be a problem.

Yet the opportunity to observe a community with different genetic make up and with a different culture and habits in varying degrees of transition to the Western model provides investigators with a unique opportunity. Descriptive studies such as this, which use systematically collected health records, provide a starting point.

\section{References}

${ }^{1}$ Clarke M, Samani N, Diamond P. Tuberculosis morbidity amongst immigrants: notification and hospitalisation. Community Med 1979;1: 23-8.

${ }^{2}$ Stanfield JP, Reid D. Imported infections in children. $\mathcal{F} R$ Coll Physicians Lond 1980;14:232-7.

3 Anonymous. Exotic diets and the infant [Editorial]. Br Medf 1978; :804-5.

Anonymous. Ethnic differences in common diseases. Postgrad Med $\mathcal{F} 1981$ 57:745-93.

${ }^{5}$ Gordon T. Mortality experience among the Japanese in the United States, Hawaii and Japan. Public Health Rep 1957;72:543-53.

${ }^{6}$ Anonymous. Ethnic factors in disease [Editorial]. $\mathrm{Br}$ Med $\mathcal{F} 1981$;282: 1496-7.

7 Sillitoe K. Ethnic origin: the search for a question. Population Trends 1978;13:25-30.

8 Jones PR. Research report: ethnic intermarriage in Britain. Ethnic and Racial Studies $1982 ; 5: 223-8$

- Office of Population Censuses and Surveys. Monitor-1981 census. London: OPCS, 1980. (Cen 80/81.)

10 Woolf B. On estimating the relation between blood group and disease. Ann Hum Genet 1955;19:251-3.

11 World Health Organisation. Manual of the international statistical classification of diseases, injuries and causes of death. Vol 1. Geneva: WHO, 1967.

12 Marmot MG, Adelstein AM, Bulusa L. Cardiovascular mortality among immigrants to England and Wales. Postgrad Med $\mathcal{f}$ 1981;57:760-2.

13 Beevers DG, Cruickshank JK. Age, sex, ethnic origin and hospital admission for heart attack and stroke. Postgrad Med f 1981 ;57:763-5.

14 Morrison-Smith J, Cooper SM. Asthma and atopic disease in immigrants from Asia and the West Indies. Postgrad Med $\mathcal{F} 1981$;57:774-6.

15 Clarke M. The quality and organisation of medical care provided to immigrants in pregnancy in Leicestershire. In: McFadyen IR, MacVicar J, eds. Problems of the Asian community in Britain: a scientific meeting of the Royal College of Obstetricians and Gynaecologists. London: Royal College of Obstetricians and Gynaecologists, 1982:109-16.

(Accepted 13 fanuary 1983)

Any intake of the major energy-yielding nutrients (carbohydrate, protein, or fat) in excess of a person's energy requirements will usually be stored as body fat.-D A T southgate, head, Division of Nutrition and Food Quality, Norwich.

Is there any risk to health from a 30 year old cast iron cooker that is insulated with asbestos and in regular use?

As the insulation for the cooker is not on the external surface and not liable to abrasion, there are no risks to health from using the cooker. If, however, the cooker was shaken by movement then some free asbestos fibres may be released. They should be removed by a vacuum cleaner and not swept up. If the cooker were to be dismantled for repair things would be very different. Any bonding holding the asbestos fibres would almost certainly be broken down by 30 years of periods at high temperature and quantities of dust could be released into the atmosphere. Furthermore, quite possibly blue asbestos (crocidolite) was used in the manufacture in the early 1950s. Repair

work should be undertaken only under circumstances where the nature of the fibres could be determined and suitable precautions taken.-W R LEE, professor of occupational health, Manchester.

Should there be any limit to the number of marathons a person should run in any one year? Will running do varicose veins any harm?

Although most marathon runners prefer to run a marathon only three or four times a year, some enthusiasts run as many as one a month and there is even one enthusiast who runs one every weekend. Provided that they do not become severely dehydrated, and so risk kidney damage or heat stroke, there is no physiological reason why, if they enjoy running frequent marathons, they should not do so. Many athletes have varicose veins and vigorous exercise does not usually cause problems and, indeed, may improve the nutrition of the legs as the calf pump is being used to increase venous return to the heart. Obviously there is a small risk of trauma to the veins. - D s TUNSTALL-PEDOE, consultant cardiologist, and medical director of the London Marathon. 